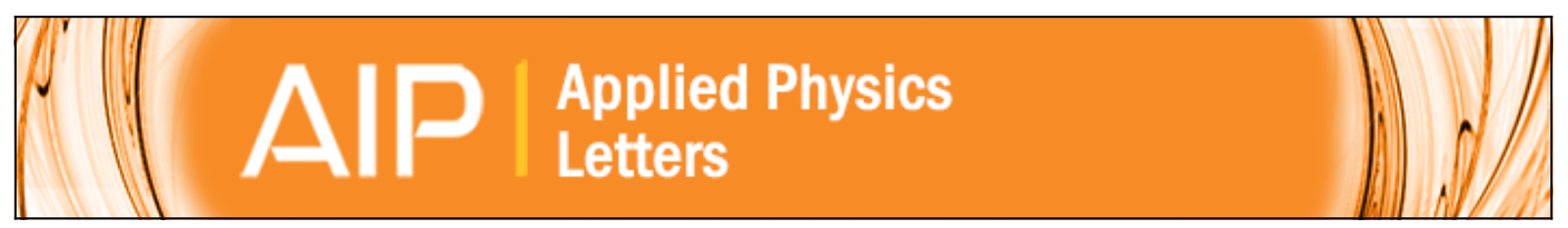

\title{
Thermal rectification of electrons in hybrid normal metal-superconductor nanojunctions
}

\author{
F. Giazotto and F. S. Bergeret
}

Citation: Applied Physics Letters 103, 242602 (2013); doi: 10.1063/1.4846375

View online: http://dx.doi.org/10.1063/1.4846375

View Table of Contents: http://scitation.aip.org/content/aip/journal/apl/103/24?ver=pdfcov

Published by the AIP Publishing

\section{Articles you may be interested in}

Influence of interface transmissivity and inelastic scattering on the electronic entropy and specific heat of diffusive superconductor-normal metal-superconductor Josephson junctions

J. Appl. Phys. 105, 093904 (2009); 10.1063/1.3121210

Erratum: Temperature dependence of superconductor-correlated metal-superconductor Josephson junctions [Appl. Phys. Lett. 82, 970 (2003)]

Appl. Phys. Lett. 83, 1275 (2003); 10.1063/1.1600849

High-transparency superconductor-insulator-normal- metal-insulator-superconductor Josephson junctions for digital electronics

Appl. Phys. Lett. 81, 1273 (2002); 10.1063/1.1499768

Direct writing of low T c superconductor-normal metal-superconductor junctions using a focused ion beam Appl. Phys. Lett. 75, 262 (1999); 10.1063/1.124342

Superconductor-normal metal-superconductor Josephson junctions with Ti interlayer Appl. Phys. Lett. 73, 1583 (1998); 10.1063/1.122211

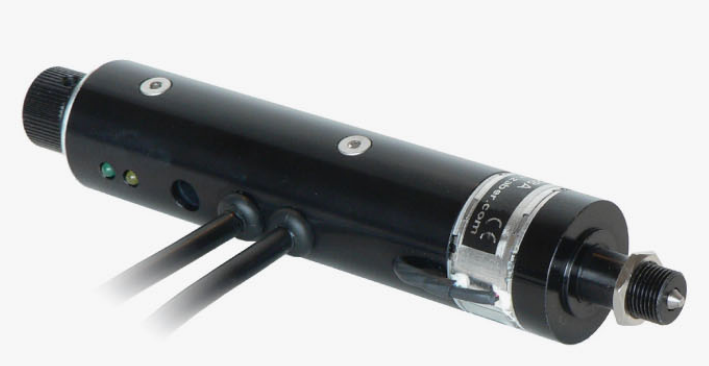

Automate your set-up with 


\title{
Thermal rectification of electrons in hybrid normal metal-superconductor nanojunctions
}

\author{
F. Giazotto ${ }^{1, a)}$ and F. S. Bergeret ${ }^{2,3, b)}$ \\ ${ }^{1}$ NEST, Instituto Nanoscienze-CNR and Scuola Normale Superiore, I-56127 Pisa, Italy \\ ${ }^{2}$ Centro de Física de Materiales (CFM-MPC), Centro Mixto CSIC-UPV/EHU, Manuel de Lardizabal 4, \\ E-20018 San Sebastián, Spain \\ ${ }^{3}$ Donostia International Physics Center (DIPC), Manuel de Lardizabal 5, E-20018 San Sebastián, Spain
}

(Received 15 October 2013; accepted 25 November 2013; published online 13 December 2013)

\begin{abstract}
We theoretically investigate heat transport in hybrid normal metal-superconductor (NS) nanojunctions focusing on the effect of thermal rectification. We show that the heat diode effect in the junction strongly depends on the transmissivity and the nature of the NS contact. Thermal rectification efficiency can reach up to $\sim 123 \%$ for a fully transmissive ballistic junction and up to $84 \%$ in diffusive NS contacts. Both values exceed the rectification efficiency of a NIS tunnel junction (I stands for an insulator) by a factor close to $\sim 5$ and $\sim 3$, respectively. Furthermore, we show that for NS point-contacts with low transmissivity, inversion of the heat diode effect can take place. Our results could prove useful for tailoring heat management at the nanoscale, and for mastering thermal flux propagation in low-temperature caloritronic nanocircuitry. (C) 2013 AIP Publishing LLC. [http://dx.doi.org/10.1063/1.4846375]
\end{abstract}

Control of the heat flow at the nanoscale has been attracting the attention of several research groups in the last decade. $^{1,2}$ An accurate understanding of heat transport is essential, for instance, for a fine control of ultrasensitive cryogenic radiation detectors, ${ }^{1,3}$ nanocoolers,${ }^{1,4}$ and caloritronic circuits. ${ }^{5-11}$ In several cases, such devices contain superconductors as building block elements which introduce phase coherence to the heat transport. Examples include Josephson heat interferometers ${ }^{12}$ and thermal quantum diffractors ${ }^{13}$ in which the heat current is controlled by a magnetic flux, or electronic refrigeration in normal metal-superconductor $(\mathrm{NS})^{1}$ and ferromagnet-superconductor $(\mathrm{FS})^{14,15}$ structures whose efficiency depends on Andreev reflection ${ }^{16}$ at the interface with the superconductor.

In a voltage-biased NS junction, the charge current consists of two contributions: the quasiparticle and the Andreev current. ${ }^{16}$ For voltages $V$ below the superconducting energy gap, the latter may dominate, and the amplitude of the current depends on the transmissivity and the nature of the contact. Due to the electron-hole symmetry and for a spatially symmetric barrier at the SN interface, the amplitude of the electric current does not depend on the sign of $V$. The same holds as well for the heat current flowing through the junction in a voltage-biased configuration. ${ }^{1}$ By contrast, the electronic contribution to the heat current in the presence of a temperature bias across the NS junction depends on the sign of the temperature drop. ${ }^{17}$ This property stems from the strong temperature dependence of the superconducting density of states at high temperatures. In this regard, a NS junction therefore behaves as a thermal diode ${ }^{18,19}$ with this meaning that heat conduction along one direction is preferred with respect to that occurring upon temperature bias reversal. Strong effort has been devoted so far to envision

\footnotetext{
${ }^{\text {a)} E l e c t r o n i c ~ m a i l: ~ g i a z o t t o @ s n s . i t ~}$

b)Electronic mail: sebastian_bergeret@ehu.es
}

and to realize thermal rectifiers dealing, for instance, either with phonons, ${ }^{20-24}$ electrons, ${ }^{17,25-29}$ or with photons. ${ }^{30}$

In this letter, we address the heat diode effect in NS nanojunctions and explore how thermal rectification depends on the interface properties. We show that a perfectly transparent point contact can provide a large rectification coefficient up to $\sim 123 \%$ which exceeds by a factor close to 5 the one predicted to occur in NIS tunnel junctions. ${ }^{17}$ In more realistic diffusive junctions, the maximum heat rectification efficiency can be as large as $\sim 84 \%$. Furthermore, in a NS point-contact thermal rectification can change sign as a function of temperature for a low enough interface transmissivity. Our predictions for the heat diode effect in hybrid NS junctions could prove useful for developing future caloritronic nanodevices.

The system under investigation is schematized in Fig. 1 and consists of a temperature-biased NS junction. The electronic temperature in $\mathrm{N}$ and $\mathrm{S}$ is set to $T_{N}$ and $T_{S}$, respectively. We assume a spatially uniform temperature in the electrodes so to avoid the generation of any thermal gradient within each of them. In the forward thermal bias configuration [Fig. 1(a)], a thermal gradient is intentionally created developing at the NS interface by setting $T_{N}=T_{h o t}>T_{S}$ $=T_{\text {cold }}$ which gives rise to a total heat flux $\dot{Q}_{+}$through the system. By contrast, in the reverse thermal bias configuration, the thermal gradient is inverted so that $T_{N}=T_{\text {cold }}$ $<T_{S}=T_{h o t}$ which yields a total heat current $\dot{Q}_{-}$flowing from $\mathrm{S}$ to $\mathrm{N}$ [Fig. 1(b)]. We note that by definition $\dot{Q}_{+}$and $\dot{Q}_{-}$have opposite sign. The hatched circles in the figure indicate the NS contact region which, as discussed below, can be ballistis or tunnel as well as diffusive or dirty. The thermal rectification coefficient $(R)$ can be defined as ${ }^{17}$

$$
R(\%)=\frac{\left|\dot{Q}_{-}\right|-\dot{Q}_{+}}{\dot{Q}_{+}} \times 100 .
$$

According to Eq. (1), $R=0$ implies the absence of a heat rectification whereas $R>0$ implies a thermal current 


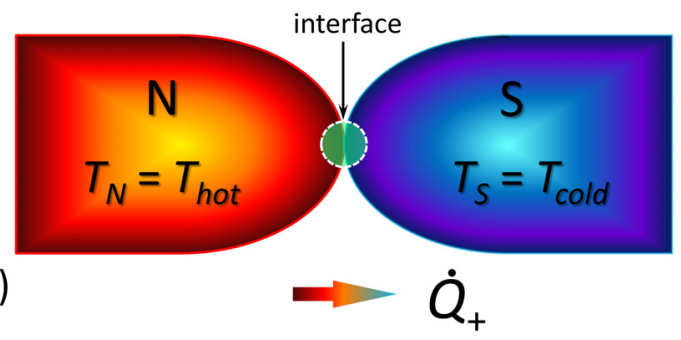

(a)

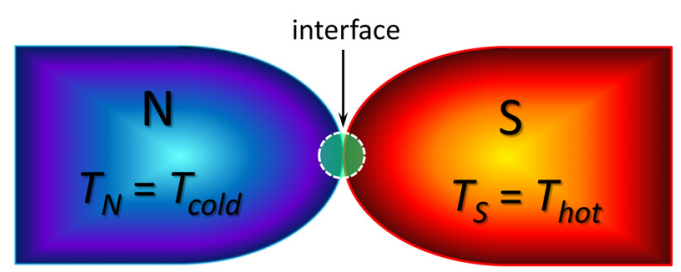

(b)

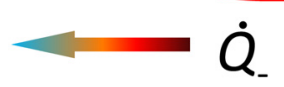

FIG. 1. Scheme of a hybrid NS heat diode under forward (a), and reverse (b) thermal bias configuration. The NS junction is temperature-biased with $T_{N} \neq T_{S}$, and $\dot{Q}_{+}$and $\dot{Q}_{-}$denote the heat current flowing through the structure in the forward $\left(T_{N}>T_{S}\right)$ and reverse $\left(T_{N}<T_{S}\right)$ thermal-bias setup, respectively. The circular hatched regions indicates the NS interface which can describe a ballistic or tunnel junction as well as a diffusive or dirty contact.

flowing preferentially from the $\mathrm{S}$ toward the $\mathrm{N}$ side of the junction.

For a quantitative description of the charge and heat transport through the NS junction, it is convenient to introduce the Keldysh Green's functions

$$
\check{G}_{S(N)}=\left(\begin{array}{cc}
\hat{G}^{R} & \hat{G}^{K} \\
0 & G^{A}
\end{array}\right),
$$

where the retarded $(\mathrm{R})$, advanced (A), and Keldysh components in the $\mathrm{S}$ and $\mathrm{N}$ electrodes are given by

$$
\begin{gathered}
\hat{G}_{N}^{R(A)}= \pm \tau_{3}, \\
\hat{G}_{N}^{K}=2 \tau_{3} \tanh \left(\frac{E}{2 k_{B} T_{N}}\right), \\
\hat{G}_{S}^{R(A)}=g^{R(A)} \tau_{3}+f^{R(A)} i \tau_{2}, \\
\hat{G}_{S}^{K}=\left(\hat{G}_{S}^{R}-\hat{G}_{S}^{A}\right) \tanh \left(\frac{E}{2 k_{B} T_{S}}\right) .
\end{gathered}
$$

In the above expressions, $\tau_{i}$ are the Pauli matrices in the Nambu space, $\quad g^{R(A)}=\left(E / \Delta\left(T_{S}\right)\right) f^{R(A)}=E / \xi^{R(A)}, \xi^{R(A)}$ $=\sqrt{(E \pm i \eta)^{2}-\Delta^{2}\left(T_{S}\right)}, \Delta\left(T_{S}\right)$ is the BCS temperaturedependent superconducting order parameter, $T_{N(S)}$ is the temperature of the $\mathrm{N}(\mathrm{S})$ electrode, and $k_{B}$ is the Boltzmann constant. The parameter $\eta$ accounts for the inelastic scattering rate within the relaxation time approximation. ${ }^{31,32}$

The electronic transport through the NS junction can be described using the matrix current $(\hat{I})$ introduced by Nazarov, ${ }^{33}$

$$
\hat{I}=-\frac{2 e^{2}}{\pi \hbar} \sum_{n} \tau_{n}\left[\check{G}_{S}, \check{G}_{N}\right]\left[4-\tau_{n}\left(2-\left\{\check{G}_{N}, \check{G}_{S}\right\}\right)\right]^{-1}
$$

Here, $\tau_{n}$ is the transmission of the $n$th junction channel, and the sum goes over the junction conducting channels. In our analysis, we shall focus on the electronic contribution to the heat current only, $\dot{Q}$, which is defined as

$$
\dot{Q}=\frac{1}{8 e^{2}} \int_{-\infty}^{\infty} E \operatorname{Tr} \hat{I}^{K} \mathrm{~d} E,
$$

and we do not take into account neither the heat exchanged between electrons and phonons nor a pure phononic heat current. ${ }^{5,12}$ From Eqs. (3) to (8), we get for $T_{N} \neq T_{S}$ the following expression for the heat current flowing through the contact

$$
\begin{aligned}
\dot{Q}= & \frac{1}{2 \pi \hbar} \sum_{n} \int_{-\infty}^{\infty} \mathrm{d} E E \frac{\tau_{n}}{2-\tau_{n}\left(1+g^{A}\right)} \\
& \times\left[\left(g^{R}-g^{A}\right)-\frac{2 \tau_{n}\left(f^{R}-f^{A}\right) f^{R}}{4-2 \tau_{n}\left(1-g^{R}\right)}\right] \\
& \times\left[\tanh \left(\frac{E}{2 k_{B} T_{S}}\right)-\tanh \left(\frac{E}{2 k_{B} T_{N}}\right)\right] .
\end{aligned}
$$

In our notation, $\dot{Q}>0$ represents the heat current flowing out of the $\mathrm{N}$ lead when $T_{N}>T_{S}$. Equation (9) is a general expression that describes the heat flow for an arbitrary contact. It is worth noting that the values of the transmission coefficients $\tau_{n}$ in Eqs. (7)-(9) are those in the normal state. These coefficients can be obtained from microscopic models for an accurate description of a realistic interface by means of $a b$ initio calculations (see, for instance, Ref. 2 and references therein). Here, instead we analyze a wide range of interfaces described by certain distribution of channel's transmissions.

For example, a point-contact is defined by a unique conducting channel with transmission $\tau$. A ballistic junction is described by setting all channel transmissions $\tau_{n}=1$, whereas in the case of a tunnel contact all $\tau_{n} \ll 1$. In the latter case, from Eq. (8), we recover the well-known expression for the heat current $\left(\dot{Q}_{\text {tunnel }}\right)$ flowing through a temperaturebiased superconducting tunnel junction, ${ }^{1}$ i.e.,

$$
\begin{aligned}
\dot{Q}_{\text {tunnel }}= & \frac{G_{N}}{e^{2}} \int_{\Delta\left(T_{S}\right)}^{\infty} \mathrm{d} E \frac{E^{2}}{\sqrt{E^{2}-\Delta\left(T_{S}\right)^{2}}} \\
& \times\left[\tanh \left(\frac{E}{2 k_{B} T_{S}}\right)-\tanh \left(\frac{E}{2 k_{B} T_{N}}\right)\right],
\end{aligned}
$$

where $G_{N}=\left(e^{2} / \pi \hbar\right) \sum_{n} \tau_{n}$ is the contact normal-state conductance.

In the case of an extended NS interface with a continuous distribution of channels, one can replace in Eq. (9) the sum $\sum_{n}$ with the integral $\int_{0}^{1} d \tau \mathcal{P}(\tau)$, where $\mathcal{P}(\tau)$ is the interface transmission distribution function. Realistic interfaces between metals are typically dirty and can be described by a scattering region of a certain characteristic length. If this length is larger than the Fermi wave length, the interface is 
called to be diffusive and is characterized by the following distribution function: ${ }^{34}$

$$
\mathcal{P}(\tau)=\frac{\hbar G_{N}}{2 e^{2}} \frac{1}{\tau \sqrt{1-\tau}} .
$$

By contrast, if the characteristic scattering region is much smaller than the Fermi wave length (i.e., a sharp interface), the distribution function reads ${ }^{35}$

$$
\mathcal{P}(\tau)=\frac{\hbar G_{N}}{e^{2}} \frac{1}{\tau^{3 / 2} \sqrt{1-\tau}} .
$$

Thus, with the help of Eqs. (9)-(12) we are able to describe heat transport through a large variety of junctions and obtain the thermal rectification coefficient $R$. In the normal state, i.e., for temperatures larger than the superconducting critical one, $\Delta=0$ and Eq. (9) reduces to

$$
\dot{Q}=\frac{k_{B}^{2} G_{N} \pi^{2}}{6 e^{2}}\left(T_{N}^{2}-T_{S}^{2}\right) .
$$

This expression shows that no thermal rectification occurs in a full normal-metal junction.

We are now able to explore the thermal diode properties of the NS contact by calculating the rectification coefficient [see Eq. (1)]. To this end, it is illustrative to start our discussion considering first the heat rectification characteristics of a point-contact ballistic NS junction characterized by a unique channel of transmission $\tau$. Figure 2(a) shows the rectification efficiency $R$ vs $T_{\text {hot }}$ for $T_{\text {cold }}=0.1 T_{c}$ and for several values of $\tau$. Above, $T_{c}=\left(1.764 k_{B}\right)^{-1} \Delta_{0}$ is the superconducting critical temperature while $\Delta_{0}$ is the zerotemperature energy gap. In general, for any transmission, $R$ is a non-monotonic function of the temperature peaked at a specific $T_{h o t}$ which depends on $\tau$, then rapidly decreasing at higher temperature. In particular, for a perfect transmissive interface $(\tau=1)$ a maximum thermal rectification coefficient as high as $\sim 123 \%$ is obtained at $T_{\text {hot }}=T_{c}$. This large $R$ value stems from ideal Andreev reflection ${ }^{16}$ at the NS interface. For $\tau \gtrsim 0.1$, the heat rectification turns out to be always positive in the whole range of temperatures. By reducing $\tau$ yields a suppression of the maximum of $R$ which is attained for smaller values of $T_{h o t}$. Notably, negative $R$ values are obtained at large $T_{\text {hot }}$ temperatures, i.e., for $T_{h o t}>T_{c}$. This sign inversion of the thermal rectification coefficient implies that the heat current flows preferentially from $\mathrm{N}$ to $\mathrm{S}$. For low interface transmissivity (i.e., $\tau=10^{-4}$ ), which describes a NIS tunnel junction, $R$ reaches values as large as $\sim 26 \%$ at $T_{\text {hot }} \simeq 0.85 T_{c} .{ }^{17} \mathrm{We}$ stress that the latter value is around $\sim 20 \%$ of the maximum reached in the $\tau=1$ limit. It is worthwhile to mention that thermal rectification is a fully non linear effect, and that it is absent in the linear response regime. The dependence of the maximum thermal rectification efficiency $\left(R_{\max }\right)$ as a function of the transmission coefficient for a point-contact is shown in Fig. 2(b). In particular, the plot shows that for $\tau=0.5$ thermal rectification is reduced by almost a factor of two with respect to the ideal junction, whereas the lowest saturation limit is already reached for $\tau \lesssim 10^{-3}$.
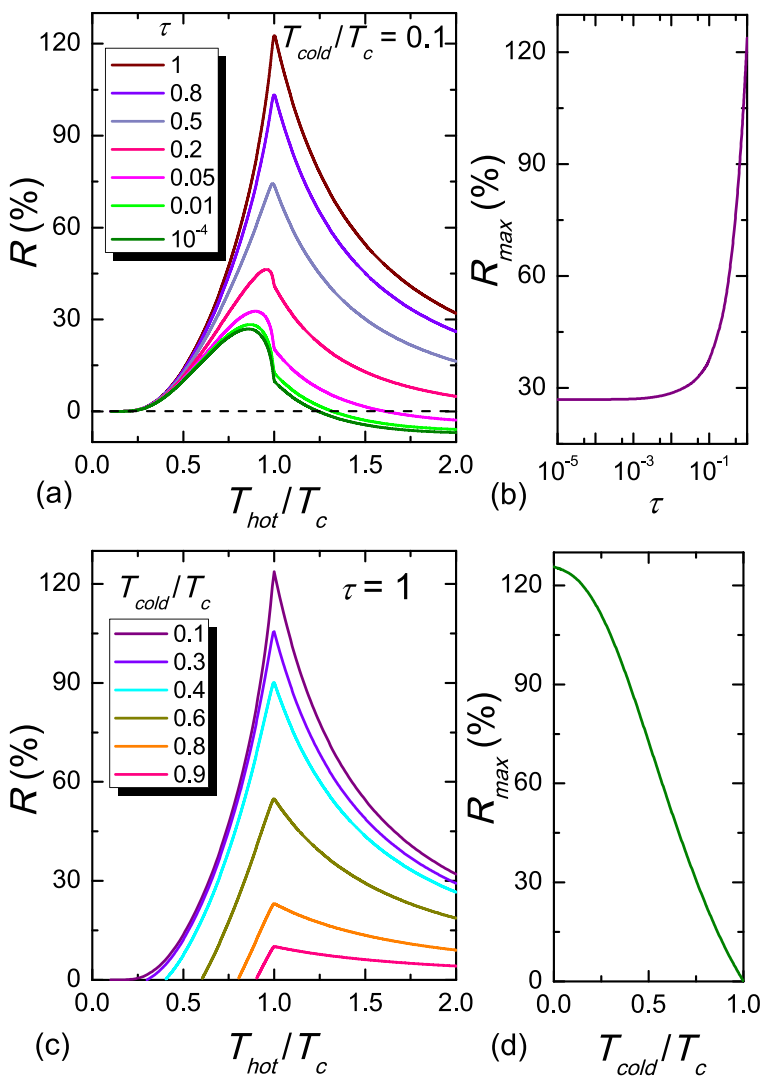

FIG. 2. (a) Rectification efficiency of a point-contact NS junction $R$ vs $T_{h o t}$ calculated at $T_{\text {cold }}=0.1 T_{c}$ for several values of the transmission coefficient $\tau$. (b) Maximum thermal rectification efficiency of a ballistic junction $R_{\max }$ vs $\tau$ for $T_{\text {cold }}=0.1 T_{c}$. (c) $R$ vs $T_{\text {hot }}$ for a perfectly transmitting $(\tau=1)$ ballistic NS junction calculated for a few values of $T_{\text {cold. }}$ (d) $R_{\max }$ vs $T_{\text {cold }}$ calculated for $\tau=1 . T_{c}$ denotes the superconducting critical temperature.

The effect of the smaller temperature $T_{\text {cold }}$ onto $R$ for a perfectly transmitting NS point-contact is displayed in Fig. 2(c) as a function of $T_{\text {hot }}$. In particular, the increase of $T_{\text {cold }}$ leads to a suppression of $R$. We emphasize that the sign of thermal rectification turns out to be positive in the whole range of temperatures, while $R$ obtains its maximum values always for $T_{h o t}=T_{c}$. The evolution of the maximum rectification efficiency $R_{\max }$ with $T_{\text {cold }}$ is shown in Fig. 2(d). It can be noted how $R$ is reduced by increasing the temperature. In particular, $R$ reaches $\sim 57 \%$ of the maximum at $T_{\text {cold }}=0.5 T_{c}$.

In order to assess the full applicability of heat rectifiers based on NS junctions, we consider now less ideal hybrid contacts, i.e., NS junctions with diffusive or dirty interfaces. These are characterized by distributions of transmissivities described by Eqs. (11) or (12), respectively. Figure 3(a) shows the comparison of the thermal rectification coefficient $R$ versus $T_{\text {hot }}$ calculated at $T_{\text {cold }}=0.1 T_{c}$ for four representative different types of NS interfaces: ballistic $\left(\tau_{n}=1\right)$, diffusive, dirty and tunnel $\left(\tau_{n} \ll 1\right)$. In particular, for diffusive and dirty interfaces $R$ turns out to be always positive, with a shape strongly resembling that of the ballistic case. The maximum values for $R$ are $\sim 84 \%$ and $\sim 63 \%$ for a diffusive and dirty interface, respectively, and occur at $T_{h o t}=T_{c}$. Such a reduction of the $R$ coefficient stems from a substantial suppression of the Andreev reflection transmission occurring in diffusive or dirty contacts in comparison to the fully transmitting ballistic case. ${ }^{36}$ In spite of such a reduction, both 

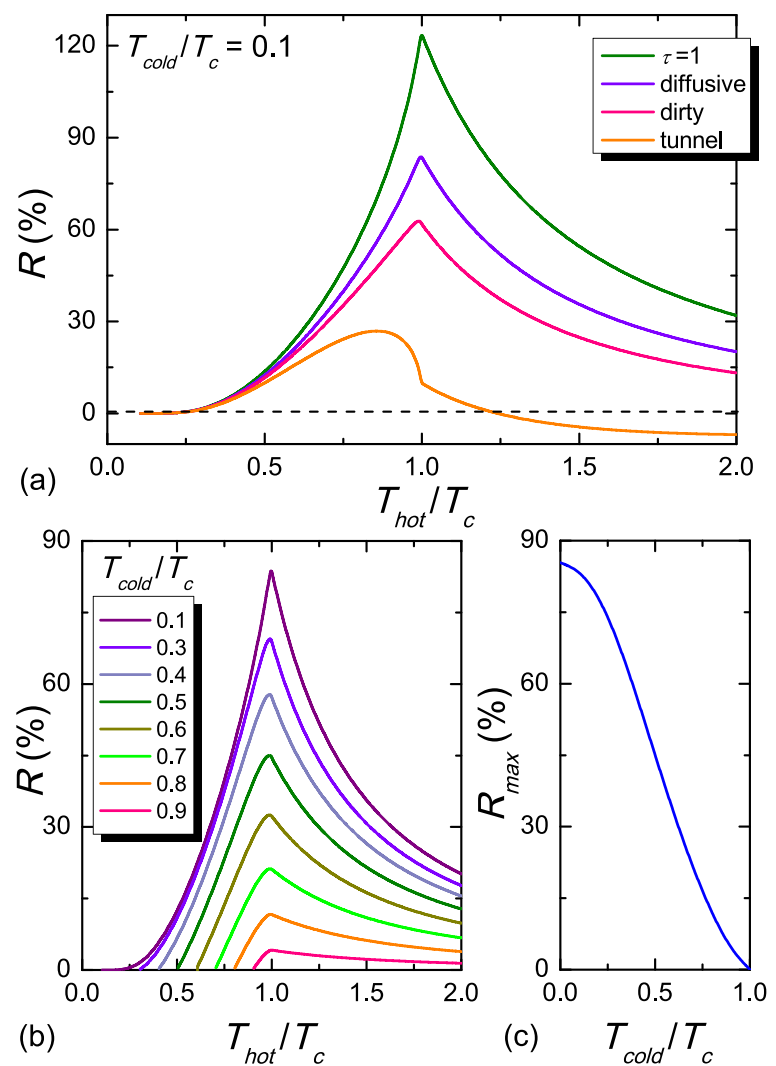

FIG. 3. (a) Comparison of thermal rectification coefficient $R$ vs $T_{\text {hot }}$ for four different types of NS junctions calculated at $T_{\text {cold }}=0.1 T_{c}$. (b) $R$ vs $T_{\text {hot }}$ for a diffusive NS junctions calculated for several values of $T_{\text {cold }}$. (c) Maximum rectification efficiency $R_{\max }$ vs $T_{\text {cold }}$ for a diffusive NS junction.

diffusive and dirty junctions are still able to provide a sizeable thermal rectification efficiency which obtains values up to factor of 3 larger than the maximum achievable with a NIS tunnel junction.

In Fig. 3(b), we show the behavior of $R$ for a diffusive NS junction calculated against $T_{\text {hot }}$ for several values of $T_{\text {cold }}$ (for dirty interfaces, similar results, not shown here, are obtained). By increasing $T_{\text {cold }}$ yields a reduction of the maximum rectification efficiency, being the sign always positive. The dependence of $R_{\max }$ on $T_{\text {cold }}$ is displayed in Fig. 3(c) and shows that at $T_{\text {cold }}=0.5 T_{c}$ the coefficient $R$ can obtain values as large as the $\sim 54 \%$ of the maximum achievable. The behavior described above for a diffusive NS contact therefore confirms the picture that this kind of junctions can provide a substantially large $R$ in a wide range of temperatures.

From a practical point of view and in light of a realistic implementation, superconducting aluminum $(\mathrm{Al})$ or vanadium $(\mathrm{V})$ combined, for instance, with copper $(\mathrm{Cu})$ as a normal metal would allow the fabrication of diffusive NS nanojunctions. ${ }^{37-39}$ On the other side, InAs-based twodimensional electron gases combined with niobium $(\mathrm{Nb})$ would enable the realization of Schottky barrier-free highly transmissive semiconductor-superconductor ballistic junctions. ${ }^{40-42}$ These predictions for thermal rectifications could be tested experimentally in a prototype hybrid microstructure designed along the lines of that presented in Ref. 17, in particular by symmetrically tunnel-coupling two additional identical normal metal electrodes to the NS junction. Electron heating and thermometry can be performed through NIS tunnel or SNS Josephson junctions ${ }^{1}$ coupled to the $\mathrm{N}$ leads, therefore allowing to realize selectively the forward and reverse thermal-bias configuration in the structure. Concerning potential applications, NS thermal rectifiers could be exploited in the field of electronic cooling, or for thermal isolation and heat management at the nanoscale. Moreover, other caloritronic devices such as heat interferometers, sensitive radiation detectors, or magnetic sensors might likely benefit from the combination with NS thermal diodes to improve their performance.

In summary, we have theoretically analyzed thermal rectification in normal metal-superconductor nanojunctions comparing different types of NS contacts. We have shown, in particular, that by increasing the interface transmissivity leads to a substantial enhancement of the heat diode effect whereas the sign of rectification can be changed in a suitable range of temperatures for low junction transparency. For perfectly transmissive ballistic contacts, thermal rectification can be as high as $123 \%$ thus exceeding by a factor close to $\sim 5$ that is achievable in NIS tunnel junctions. For diffusive contacts, the rectification efficiency can obtain values as high as $\sim 84 \%$. Because of the above results and of the ease intrinsic in their fabrication, NS junctions appear therefore as a promising building block for the implementation of effective heat diodes to be exploited in low-temperature caloritronic nanocircuitry.

F.G. acknowledges the Italian Ministry of Defense through the PNRM project "TERASUPER," and the Marie Curie Initial Training Action (ITN) Q-NET 264034 for partial financial support. The work of F.S.B was supported by the Spanish Ministry of Economy and Competitiveness under Project FIS2011-28851-C02-02. F.S.B thanks Professor Martin Holthaus and his group for their kind hospitality at the Physics Institute of the Oldenburg University.

${ }^{1}$ F. Giazotto, T. T. Heikkilä, A. Luukanen, A. M. Savin, and J. P. Pekola, Rev. Mod. Phys. 78, 217 (2006).

${ }^{2}$ Y. Dubi and M. Di Ventra, Rev. Mod. Phys. 83, 131 (2011).

${ }^{3}$ F. Giazotto, T. T. Heikkilä, G. P. Pepe, P. Helisto, A. Luukanen, and J. P. Pekola, Appl. Phys. Lett. 92, 162507 (2008).

${ }^{4}$ J. T. Muhonen, M. Meschke, and J. P. Pekola, Rep. Prog. Phys. 75, 046501 (2012).

${ }^{5}$ K. Maki and A. Griffin, Phys. Rev. Lett. 15, 921 (1965).

${ }^{6}$ G. D. Guttman, B. Nathanson, E. Ben-Jacob, and D. J. Bergman, Phys. Rev. B 55, 3849 (1997).

${ }^{7}$ E. Zhao, T. Löfwander, and J. A. Sauls, Phys. Rev. Lett. 91, 077003 (2003).

${ }^{8}$ M. Meschke, W. Guichard, and J. P. Pekola, Nature 444, 187 (2006).

${ }^{9}$ V. Chandrasekhar, Supercond. Sci. Technol. 22, 083001 (2009).

${ }^{10}$ V. V. Ryazanov and V. V. Schmidt, Solid State Commun. 42, 733 (1982).

${ }^{11}$ P. Virtanen and T. T. Heikkilä, Appl. Phys. A 89, 625 (2007).

${ }^{12}$ F. Giazotto and M. J. Martínez-Pérez, Nature 492, 401 (2012).

${ }^{13}$ M. J. Martínez-Pérez and F. Giazotto, e-print arXiv:1310.0639.

${ }^{14}$ F. Giazotto, F. Taddei, R. Fazio, and F. Beltram, Appl. Phys. Lett. 80, 3784 (2002).

${ }^{15}$ A. Ozaeta, A. S. Vasenko, F. W. J. Hekking, and F. S. Berger, Phys. Rev. B 85, 174518 (2012).

${ }^{16}$ A. F. Andreev, Zh. Eksp. Teor. Fiz. 46, 1823 (1964) [Sov. Phys. JETP 19, 1228 (1964)].

${ }^{17}$ M. J. Martínez-Pérez and F. Giazotto, Appl. Phys. Lett. 102, 182602 (2013).

${ }^{18}$ N. A. Roberts and D. G. Walker, Int. J. Therm. Sci. 50, 648 (2011).

${ }^{19} \mathrm{G}$. Casati, Nat. Nanotechnol. 2, 23 (2007).

${ }^{20}$ L.-A. Wu and D. Segal, Phys. Rev. Lett. 102, 095503 (2009).

${ }^{21}$ D. Segal, Phys. Rev. Lett. 100, 105901 (2008). 
${ }^{22}$ B. Li, L. Wang, and G. Casati, Appl. Phys. Lett. 88, 143501 (2006).

${ }^{23}$ M. Terraneo, M. Peyrard, and G. Casati, Phys. Rev. Lett. 88, 094302 (2002).

${ }^{24}$ C. W. Chang, D. Okawa, A. Majumdar, and A. Zettl, Science 314, 1121 (2006).

${ }^{25}$ J. Ren and J.-X. Zhu, Phys. Rev. B 87, 165121 (2013).

${ }^{26}$ T. Ruokola and T. Ojanen, Phys. Rev. B 83, 241404 (2011).

${ }^{27}$ D. M. T. Kuo and Y. C. Chang, Phys. Rev. B 81, 205321 (2010).

${ }^{28}$ T. Ruokola, T. Ojanen, and A.-P. Jauho, Phys. Rev. B 79, 144306 (2009).

${ }^{29}$ X.-O. Chen, B. Dong, and X.-L. Lei, Chin. Phys. Lett. 25, 3032 (2008).

${ }^{30}$ P. Ben-Abdallah and S.-A. Biehs, Appl. Phys. Lett. 103, 191907 (2013).

${ }^{31}$ R. C. Dynes, J. P. Garno, G. B. Hertel, and T. P. Orlando, Phys. Rev. Lett. 53, 2437 (1984).

${ }^{32}$ J. P. Pekola, T. T. Heikkilä, A. M. Savin, J. T. Flyktman, F. Giazotto, and F. W. J. Hekking, Phys. Rev. Lett. 92, 056804 (2004).

${ }^{33}$ Yu. V. Nazarov, Superlattices Microstruct. 25, 1221 (1999).
${ }^{34}$ O. N. Dorokhov, JETP Lett. 36, 318 (1982).

${ }^{35}$ K. M. Schep and G. E. W. Bauer, Phys. Rev. Lett. 78, 3015(1997).

${ }^{36}$ W. Belzig, A. Brataas, Yu. V. Nazarov, and G. E. W. Bauer, Phys. Rev. B 62, 9726 (2000).

${ }^{37}$ A. Ronzani, M. Baillergeau, C. Altimiras, and F. Giazotto, Appl. Phys. Lett. 103, 052603 (2013).

${ }^{38}$ C. P. Garcia and F. Giazotto, Appl. Phys. Lett. 94, 132508 (2009).

${ }^{39}$ H. Courtois, M. Meschke, J. Peltonen, and J. Pekola, Phys. Rev. Lett. 101, 067002 (2008).

${ }^{40}$ M. Amado, A. Fornieri, F. Carillo, G. Biasiol, L. Sorba, V. Pellegrini, and F. Giazotto, Phys. Rev. B 87, 134506 (2013).

${ }^{41}$ F. Carillo, G. Biasiol, D. Frustaglia, F. Giazotto, L. Sorba, and F. Beltram, Physica E 32, 53 (2006).

${ }^{42}$ F. Giazotto, K. Grove-Rasmussen, R. Fazio, F. Beltram, E. H. Linfield, and D. A. Ritchie, J. Supercond. 17, 317 (2004). 